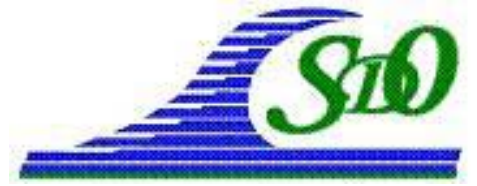

\title{
Évaluation du gain de durabilité apporté par l'utilisation d'un procédé de nappe de coffrage drainante pour un béton en site maritime
}

\author{
Benoit THAUVIN ${ }^{1}$, Nicolas ROUXEL ${ }^{1}$, Stéphane PASQUIET ${ }^{1}$
}

1. CETE de l'Ouest, Laboratoire Régional des Ponts et Chaussées de St Brieuc, Equipe de Recherche Associée au CETMEF "Durabilité des Infrastructures Portuaires et Maritimes", 5 rue Jules Vallès, 22015 Saint Brieuc Cedex, France.

benoit.thauvin@developpement-durable.gouv.fr

nicolas.rouxel@developpement-durable.gouv.fr

stephane.pasquiet@developpement-durable.gouv.fr

\section{Résumé :}

L'objectif d'une nappe de coffrage drainante (ou nappe de coffrage à perméabilité contrôlée) est d'améliorer les caractéristiques de la peau du béton. Dans la pratique, le procédé est mis en œuvre sur les panneaux de coffrage préalablement au coulage du béton. En augmentant la compacité superficielle du béton par drainage de l'eau en excès, ce procédé doit améliorer la durabilité du béton armé vis-à-vis de la corrosion des armatures. L'utilisation d'un tel procédé pourrait donc permettre d'augmenter la durée de vie des structures maritimes en béton armé en améliorant les propriétés de peau du béton et en ralentissant la pénétration des chlorures à l'origine de la corrosion.

La présente étude, réalisée à la demande du CETMEF, a pour objectif de qualifier et de quantifier le gain de durabilité apporté par l'utilisation d'une nappe de coffrage drainante. Elle s'appuie sur l'évaluation d'indicateurs de durabilité et de témoins de durée de vie. L'expérimentation repose sur la réalisation d'essais de caractérisation sur des corps d'épreuves confectionnés en utilisant un procédé de nappe de coffrage à perméabilité contrôlée.

Un dispositif dit de "vieillissement accéléré" par cycle de marnage artificiel en piscine a ainsi été conçu pour accélérer la pénétration des chlorures et ainsi évaluer l'impact du procédé de nappe de coffrage sur ce processus de pénétration. L'expérimentation a duré 454 jours. Le système de marnage a permis d'accélérer le phénomène de pénétration des chlorures. Au terme de l'expérimentation, on a observé des concentrations en chlorures plus faibles dans l'enrobage des corps d'épreuves réalisés avec le procédé de nappe de coffrage drainante. L'étude montre aussi que l'utilisation d'un tel procédé a une influence sensible sur les indicateurs de durabilité et les témoins de durée de vie. L'ensemble des résultats tendent à montrer que l'impact du procédé sur les propriétés du béton concerne l'enrobage sur une profondeur comprise entre 1 et $2 \mathrm{~cm}$

Mots-clés :

Nappe de coffrage - Corrosion - Enrobage - Durabilité - Vieillissement accéléré 


\section{Introduction}

Dans un élément en béton armé, l'une des fonctions majeures de l'enrobage est d'assurer la protection des armatures contre les agents agressifs de l'environnement extérieur qui sont notamment à l'origine du phénomène de corrosion. Indépendamment de sa formulation, la mise en œuvre du béton a une influence primordiale sur la qualité de cette couche protectrice. Plusieurs phénomènes peuvent ainsi être à l'origine de caractéristiques médiocres de l'enrobage : excès d'eau, ressuage le long du coffrage, cure insuffisante par exemple.

L'objectif d'une nappe de coffrage à perméabilité contrôlée est d'améliorer les caractéristiques de la peau du béton. En réduisant la porosité superficielle du béton, ce procédé doit améliorer les propriétés de transfert du béton d'enrobage (résistance à la pénétration du dioxyde de carbone et des chlorures) et doit donc améliorer la durabilité du béton armé vis à vis de la corrosion des armatures.

Les structures en béton armé en environnement maritime sont particulièrement exposées au risque de corrosion de leurs armatures due à la pénétration des chlorures dans le béton d'enrobage. L'utilisation d'un tel procédé pourrait donc permettre d'augmenter la durée de vie des structures en béton armé vis-à-vis de la corrosion des armatures. La présente étude a pour objectif de qualifier et de quantifier le gain de durabilité apporté par ce type de procédé. Elle s'appuie sur l'évaluation d'indicateurs de durabilité et de témoins de durée de vie conformément à l'approche performantielle (AFGC, 2004).

\section{Matériel et méthodes}

\subsection{Choix de la nappe de coffrage drainante}

Une nappe de coffrage à perméabilité contrôlée est en général constituée d'une membrane drainante en géotextile. Cette dernière est mise en place sur le coffrage préalablement au bétonnage. Elle comprend deux faces ayant des fonctions distinctes : une face "drainante" qui collecte l'air et l'eau et permet leur évacuation à l'interface avec le coffrage et une face "filtrante" qui retient les particules fines (liant essentiellement) à la surface du béton.

Avec les coffrages traditionnels, lors du serrage du béton (par vibration), l'air occlus et l'excédent d'eau migrent et s'accumulent contre la paroi du coffrage. Il en résulte un béton d'enrobage ayant un rapport E/C plus élevé que celui escompté et donc une durabilité plus faible (moins bonne compacité).

L'objectif des coffrages revêtus d'une nappe de coffrage drainante est d'évacuer l'excédent d'eau et l'air occlus dans le béton frais tout en retenant les éléments fins (ciment). In fine, le béton d'enrobage doit donc être plus compact et donc plus durable. En complément, une certaine quantité d'eau est retenue dans la nappe de coffrage. Cette eau est ré-absorbée par le béton dans les premières heures de maturation et participe donc au processus de cure. 


\section{XI $I^{\text {èes }}$ Journées Nationales Génie Côtier - Génie Civil \\ Les Sables d'Olonne, 22-25 juin 2010}
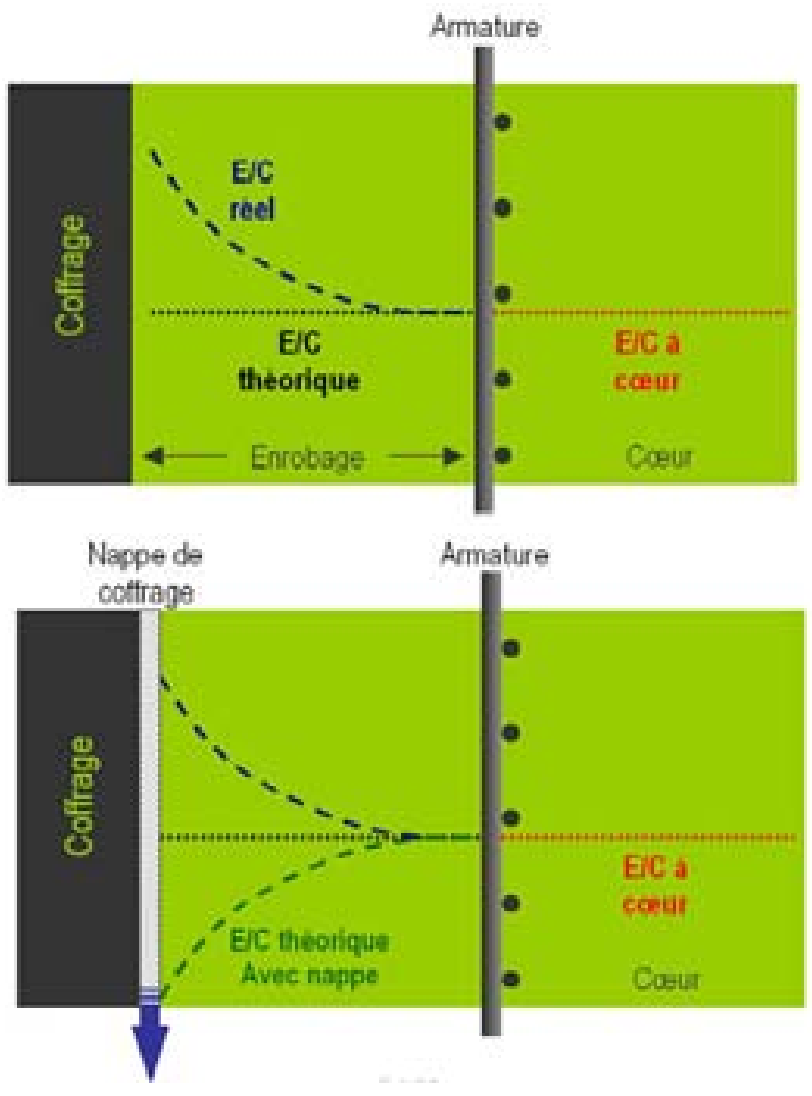

(a)

(b)

Figure 1. Principe de fonctionnement - (a) sans nappe de coffrage drainante - (b) avec nappe de coffrage drainante.

Le marché des nappes de coffrage drainantes n'est pas très développé : seuls 4 produits ont ainsi été recensés sur le marché. Ils sont basés sur le même principe : une membrane géotextile plaquée sur le coffrage assurant à la fois le drainage de l'eau et la retenue des éléments fins. L'objectif de l'étude n'étant pas de comparer les différents produits du marché, il a été décidé de sélectionner le procédé le plus couramment utilisé. Le tableau 1 en précise les principales caractéristiques.

Tableau 1. Caractéristiques de la nappe de coffrage

\begin{tabular}{|c|c|}
\hline Propriétés & $\begin{array}{l}\text { Nappe de coffrage drainante retenue dans le cadre } \\
\text { de l'étude }\end{array}$ \\
\hline Dimension moyenne des pores & $<20 \mu m$ \\
\hline Perméabilité à l'air & $<2 \mathrm{~m}^{3} / \mathrm{m}^{2}$ sec sous $98 \mathrm{~Pa}$ \\
\hline Composition & 100\% polypropylène \\
\hline Epaisseur & $0,7 \mathrm{~mm}+/-10 \%$ \\
\hline Poids unitaire & $294 \mathrm{~g} / \mathrm{m}^{2}+1-5 \%$ \\
\hline Résistance à la traction & $>18 \mathrm{kN} / \mathrm{m}$ \\
\hline Résistance à la déchirure trapézoüdale & $>500 \mathrm{~N}$ \\
\hline Drainage & Jusqu'à 3 litres d'eau par m² \\
\hline
\end{tabular}




\subsection{Les corps d'épreuve}

Il a été fait le choix de confectionner quatre dallettes en béton de $60 \mathrm{~cm}$ de hauteur, $40 \mathrm{~cm}$ de largeur et $15 \mathrm{~cm}$ d'épaisseur (tableau 2). Le nombre de dallettes a été dicté par la problématique : dallettes avec ou sans nappe de coffrage, exposition aux cycles de marnage d'eau de mer ou constamment immergées dans de l'eau douce. Leurs dimensions a été définies pour autoriser un nombre suffisant de prélèvements tout en s'affranchissant des effets de bord.

Afin d'être les plus représentatives possibles, les dallettes ont été fabriquées avec un type de béton adapté à la problématique générale de l'étude. Il s'agit d'un béton fabriqué par une centrale de béton prêt à l'emploi et livré par camion toupie dont la désignation normative est la suivante :

BPS C35/45 XC4(F) Cl0,2 Dmax 22,4 S2

CEM I 52,5N CE PMES CP2 NF (LAFARGE Le Havre)

La formule du béton était celle définie au tableau 3 .

Tableau 2. Caractéristiques principales des dallettes.

\begin{tabular}{llll}
\hline $\boldsymbol{T}$ & $\boldsymbol{C l}$ & $\boldsymbol{Z}$ & $\mathbf{Z C l}$ \\
\hline $\begin{array}{l}\text { Conservation sous } \\
\text { eau }\left(20^{\circ} \mathrm{C}\right)\end{array}$ & Cycles de marnage & $\begin{array}{l}\text { Avec nappe de coffrage } \\
\text { Conservation sous eau } \\
\left(20^{\circ} \mathrm{C}\right)\end{array}$ & $\begin{array}{l}\text { Avec nappe de coffrage } \\
\text { Cycles de marnage }\end{array}$ \\
\hline
\end{tabular}

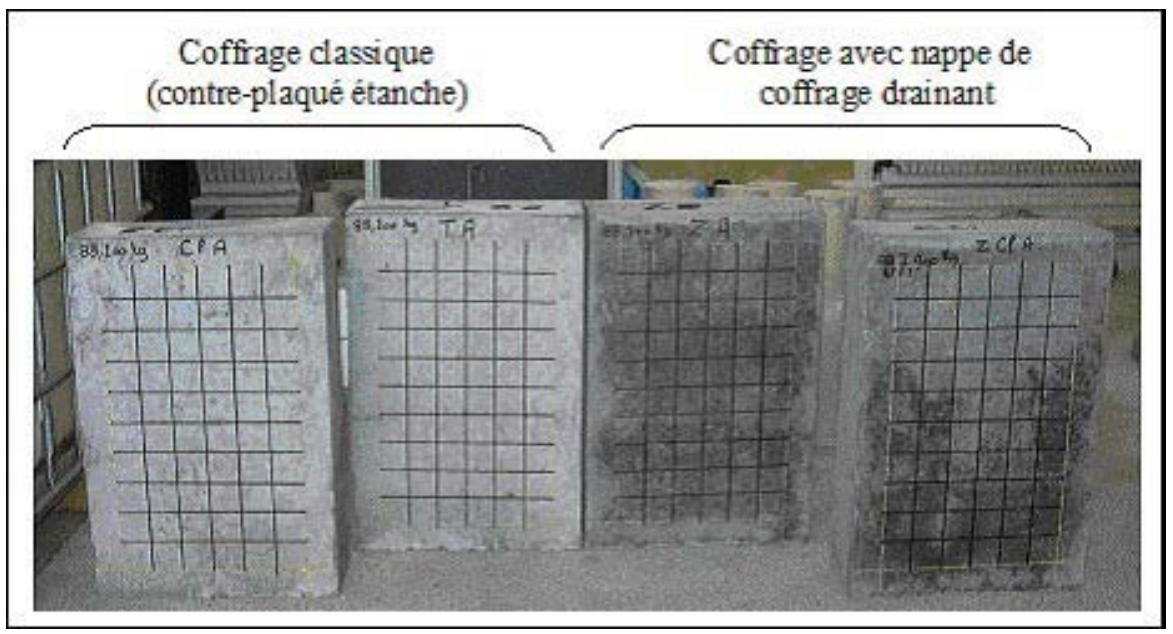

Figure 2. Dallettes de béton.

Tableau 3. Formulation du béton.

\begin{tabular}{lll}
\hline Sable & $0 / 4$ & $810 \mathrm{~kg}$ \\
Gravillon & $5 / 22,4$ & $1060 \mathrm{~kg}$ \\
Ciment & CEM I PMES & $352 \mathrm{~kg}$ \\
Adjuvant & PRE & $2,11 \mathrm{~kg}$ \\
Eau & efficace & $167 \mathrm{l}$ \\
\hline
\end{tabular}




\section{XI $I^{\grave{m} e s}$ Journées Nationales Génie Côtier - Génie Civil \\ Les Sables d'Olonne, 22-25 juin 2010}

Les caractéristiques élémentaires du béton sont celles définies au tableau 4. Le béton est conforme aux exigences réglementaires (fascicule 65). En complément, les indicateurs de durabilité du béton ont été évalués selon les principes de l'approche performantielle (AFGC, 2004), (tableau n ${ }^{\circ}$ ).

Tableau 4. Caractéristiques du béton.

\begin{tabular}{lll}
\hline Rapport eau efficace sur liant équivalent & Eeff./Leq. & 0,45 \\
Classe de chlorures & $C l \%$ & $0,12 \%$ \\
Résistance à la compression à 28 jours & $f c_{28}$ & $47,5 \mathrm{MPa}$ \\
\hline
\end{tabular}

Tableau 5. Indicateurs de durabilité et classes de durabilité potentielle.

\begin{tabular}{|c|c|c|c|}
\hline Indicateurs de durabilité & Unité & Résultat & $\begin{array}{l}\text { Classe de durabilité } \\
\text { potentielle } \\
(\text { AFGC, 2004) }\end{array}$ \\
\hline $\begin{array}{l}\text { Coefficient de diffusion des } \\
\text { chlorures }\left(D_{\text {app }}\right)\end{array}$ & $10^{-12} \mathrm{~m}^{2} \mathrm{~s}^{-1}$ & 17,2 & Faible \\
\hline $\begin{array}{l}\text { Porosité accessible à l'eau } \\
\left(P_{\text {eau }}\right)\end{array}$ & $\%$ & 10,8 & Elevée \\
\hline $\begin{array}{l}\text { Coefficient d'absorption } \\
\text { capillaire }\left(C_{a}\right)-\grave{a} 24 \text { et } 48 h\end{array}$ & kg. $\mathrm{m}^{-2}$ & $\begin{array}{l}4,32 \\
5,41\end{array}$ & Moyenne \\
\hline Résistivité électrique ( $\rho)$ & $\Omega . m$ & 69 & Faible \\
\hline
\end{tabular}

\subsection{Dispositif de vieillissement accéléré}

L'un des objectifs de l'expérimentation était de se rapprocher des conditions réelles d'exposition d'une structure en béton armé en site maritime (marnage). Or, en condition réelle, la pénétration des chlorures dans l'enrobage est un phénomène lent : de l'ordre de 30 à 50 ans pour une pénétration d'environ $4 \mathrm{~cm}$; cinétique incompatible avec les échéances visées de l'étude. Il fallait donc utiliser un dispositif permettant d'accélérer la pénétration des chlorures tout en restant le plus proche possible de la réalité.

Le dispositif dit de "vieillissement accéléré" par cycle de marnage artificiel (figures 3 et 4) a ainsi été conçu pour étudier le phénomène de corrosion due à la pénétration des chlorures dans l'enrobage. Le dispositif est destiné à accélérer la pénétration des chlorures dans les éléments en béton armé de grandes dimensions. Il permet de tester différentes formulations de béton et des techniques de réhabilitation (peintures, revêtements, inhibiteurs, etc.) ou de mise en œuvre (coffrages, etc.). Il peut également servir de calibrage à une modélisation.

Le dispositif permet d'accélérer le processus de corrosion des armatures, en favorisant la pénétration des chlorures: alternance d'immersions et de séchages, milieu agressif (eau de mer), augmentation de la fréquence du cycle de marnage. 


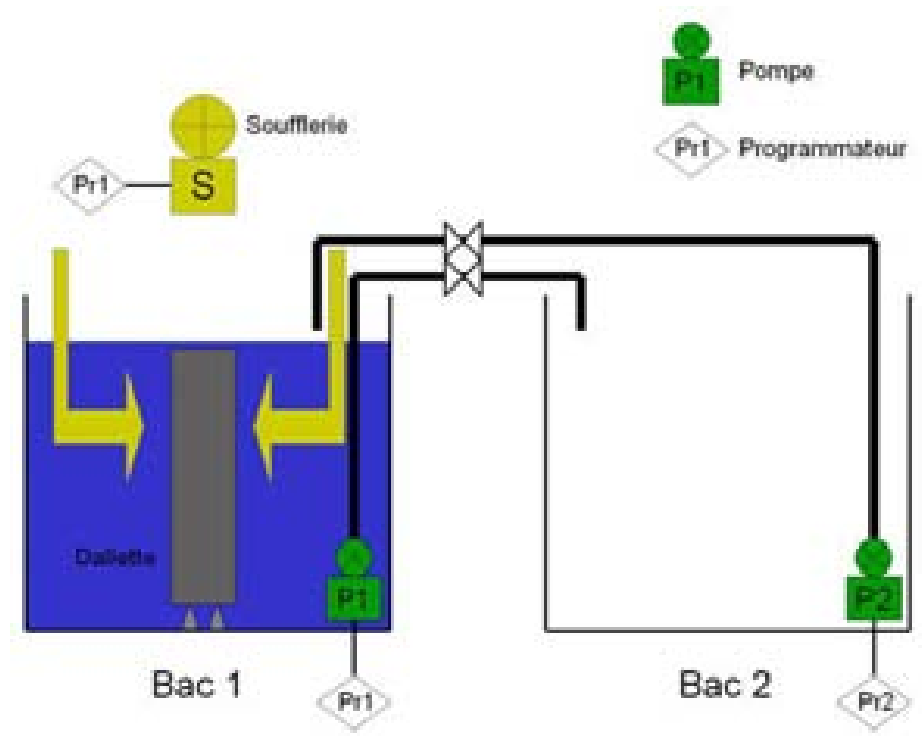

Figure 3. Dispositif en configuration "marée haute".

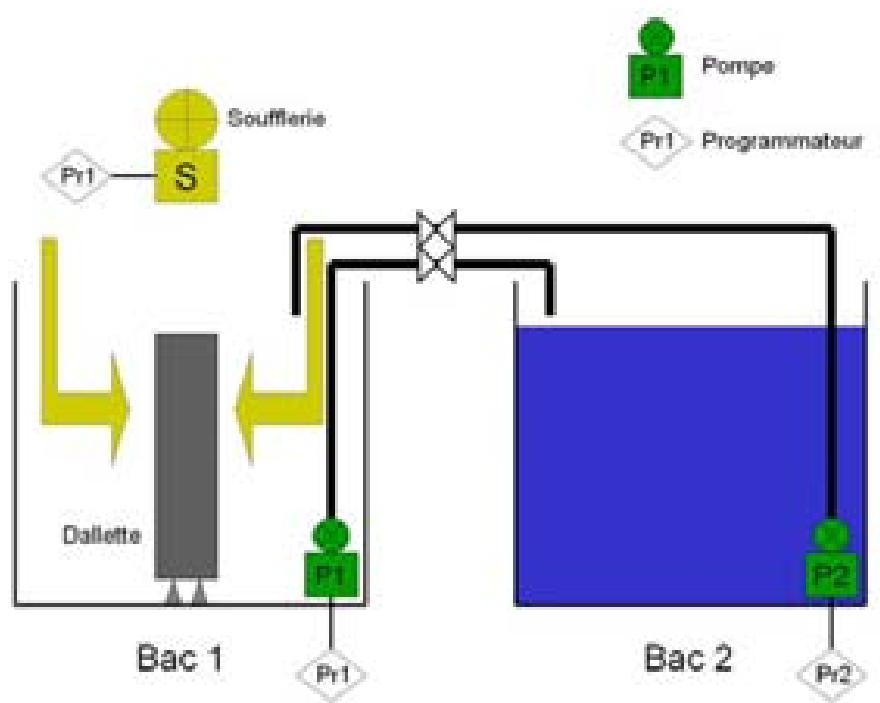

Figure 4. Dispositif en configuration "marée basse".

Le cycle présente une période de $2 \mathrm{~h}$ (figure 5) soit un cycle 6 fois plus rapide qu'un cycle de marnage naturel. L'étale de pleine mer dure 60 minutes alors que l'étale de basse mer dure 30 minutes. La transition entre ces deux phases se fait en 15 minutes. La température de d'eau est comprise entre 18 et $25^{\circ} \mathrm{C}$. Sa salinité est de $34 \mathrm{~g} / \mathrm{L}$. La soufflerie crée un vent de 1 nœud. 


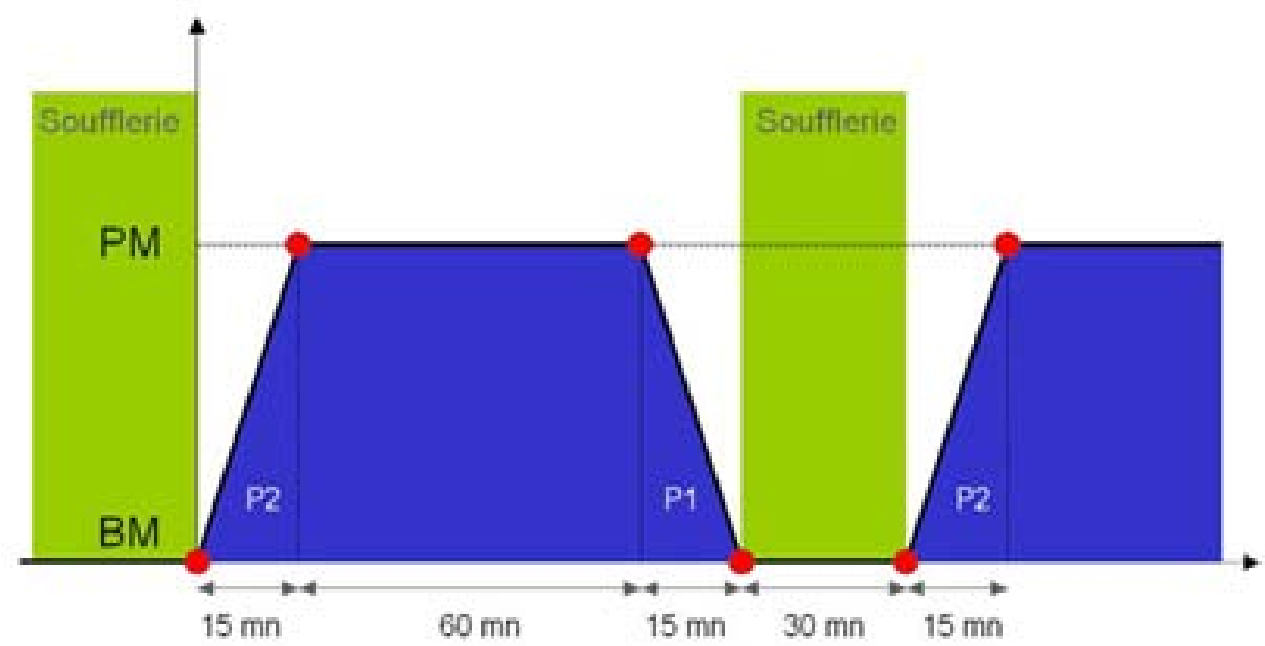

Figure 5. Cycle de marnage accéléré.

\section{Résultats}

Indépendamment des résultats présentés ci-après, il convient de noter les points suivants :

- le drainage par la nappe est effectif,

- les dallettes confectionnées avec nappes ne présentent aucun phénomène de bullage contrairement à celles confectionnées sans,

- les dallettes confectionnées avec nappes présentent une texture "rugueuse",

- après décoffrage, les dallettes confectionnées avec le dispositif drainant présentent une teinte nettement plus foncée que celles confectionnée avec un coffrage classique. Ceci peut s'expliquer par une teneur en ciment plus importante en peau pour les dallettes avec nappes. Ce phénomène s'estompe néanmoins avec le temps.

- après décoffrage, l'hétérogénéité de teinte est plus importante pour les dallettes avec nappes. Ce phénomène s'estompe néanmoins avec le temps. En fin d'expérimentation, ce sont les dallettes avec nappes qui présentent la meilleure homogénéité.

La mise en place d'une nappe drainante sur les coffrages avant le coulage du béton peut présenter un intérêt dans l'amélioration des propriétés du parement. La présente étude s'attache donc à étudier de façon qualitative et quantitative l'influence de ce dispositif sur les "indicateurs de durabilité" et les "témoins de durée de vie". Les "indicateurs de durabilité" permettent de mesurer les propriétés intrinsèques au béton étudié. Plus précisément, ces indicateurs permettent de mesurer les propriétés de transfert du béton, c'est à dire, sa capacité à résister notamment à la pénétration des chlorures. Les "témoins de durée de vie" permettent, quant à eux, de quantifier la pénétration progressive des agents agressifs (ici les chlorures) dans l'enrobage. Autrement dit, ils permettent d'évaluer l'avancement du processus de corrosion à un instant donné. La démarche 
d'évaluation adoptée s'appuie sur la méthodologie d'approche performantielle telle que décrite dans le guide AFGC (2004).

L'expérimentation comprenait la détermination de ces paramètres (indicateurs de durabilité et témoins de durée de vie) sur des prélèvements (carottes ou poudre selon les cas) effectués dans les quatre dallettes $\mathrm{T}, \mathrm{Cl}, \mathrm{Z}$ et $\mathrm{ZCl}$.

\subsection{Evaluation des indicateurs de durabilité}

Les résultats obtenus sur les carottes de 5 ou $10 \mathrm{~cm}$ d'épaisseur sont montrés au tableau 6 et ceux des carottes de $2 \mathrm{~cm}$ d'épaisseur au tableau 7 .

Tableau 6. Indicateurs de durabilité sur carottes de 5 et $10 \mathrm{~cm}$.

\begin{tabular}{|c|c|c|c|c|c|c|c|}
\hline Indicateurs de durabilité & Unité & $T$ & & $Z$ & & \multicolumn{2}{|c|}{ Ecart entre $Z$ et $T$} \\
\hline $\begin{array}{l}\text { Coefficient de diffusion des } \\
\text { chlorures }\left(D_{a p p}\right)\end{array}$ & $10^{-11} \cdot m^{2} \cdot s^{-1}$ & 13,5 & & 10,3 & & $-24 \%$ & \\
\hline $\begin{array}{l}\text { Porosité accessible à l'eau } \\
\left(P_{\text {eau }}\right)\end{array}$ & $\%$ & 11,1 & & 10,6 & & $-5 \%$ & \\
\hline $\begin{array}{l}\text { Coefficient d'absorption } \\
\text { capillaire }\left(C_{a}\right)-\grave{a} 24 \text { et } 48 h\end{array}$ & $k g \cdot m^{-2}$ & 4,76 & 5,96 & 3,32 & 4,27 & $-30 \%$ & $-28 \%$ \\
\hline Résistivité électrique $(\rho)$ & $\Omega . m$ & 62 & & 68 & & $+10 \%$ & \\
\hline Masse volumique (Mv) & kg. $\mathrm{m}^{-3}$ & 2342,5 & & 2339,1 & & $0 \%$ & \\
\hline
\end{tabular}

Tableau 7. Indicateurs de durabilité sur carottes de $2 \mathrm{~cm}$.

\begin{tabular}{lllll}
\hline Indicateurs de durabilité & Unité & $\boldsymbol{T}$ & $\boldsymbol{Z}$ & Ecart entre $\boldsymbol{Z}$ et $\boldsymbol{T}$ \\
\hline $\begin{array}{l}\text { Porosité accessible à l'eau }\left(P_{\text {eau }}\right) \\
\text { Coefficient d'absorption capillaire } \\
\left(C_{a}\right)-\text { à } 8 h\end{array}$ & $\mathrm{~kg} \cdot \mathrm{m}^{-2}$ & 1,84 & 1,61 & $-13 \%$ \\
\hline $\begin{array}{l}\text { Résistivité électrique }(\rho) \\
\text { Masse volumique }(\mathrm{Mv})\end{array}$ & $\Omega . m$ & 63 & 86 & $-13 \%$ \\
\hline
\end{tabular}

L'ensemble des indicateurs montre une amélioration des caractéristiques de durabilité du béton d'enrobage en présence de la nappe de coffrage drainante. Hormis pour le coefficient d'absorption capillaire, l'amélioration constatée sur les carottes de $2 \mathrm{~cm}$ est plus importante que sur celles de $5 / 10 \mathrm{~cm}$. La profondeur d'influence de la nappe de coffrage drainante semble se limiter aux premiers centimètres $(1 \mathrm{à} 2 \mathrm{~cm}$ ).

En complément, malgré la dispersion importante, liée en grande partie à la réalisation de la mesure, on observe une diminution sensible de la perméabilité de surface des dallettes fabriquées avec le dispositif de nappe de coffrage drainante. 
Tableau 8. Perméabilité de surface.

\begin{tabular}{lllll}
\hline Dallettes & $\begin{array}{l}\text { Temps caractéristique } \\
\text { Ti }(\text { s) } \\
\text { Moyenne }\end{array}$ & $\begin{array}{l}\text { Classe de } \\
\text { perméabilité de } \\
\text { surface }\end{array}$ & $\begin{array}{l}\text { Temps } \\
\text { caractéristique } \\
\text { Ti(s) }\end{array}$ & $\begin{array}{l}\text { Classe de } \\
\text { perméabilité de } \\
\text { surface }\end{array}$ \\
\hline$T$ & 577 & Moyenne & $<400$ & Forte \\
$Z$ & 1083 & Moyenne & $401 \grave{a} 2500$ & Moyenne \\
$C l$ & 649 & Moyenne & 2501 à 4999 & Faible \\
$\mathrm{ZCl}$ & 3617 & Faible & $>5000$ & Trèsfaible \\
\hline
\end{tabular}

\subsection{Evaluation des témoins de durée de vie}

L'étude des témoins de durée de vie s'est appuyée sur le dispositif de vieillissement accéléré. Les dallettes $\mathrm{Cl}$ et $\mathrm{ZCl}$ ont ainsi été exposées pendant 454 jours à un cycle de marnage accéléré (accélération du cycle naturel par un facteur 6). A cinq échéances, des prélèvements ont été effectués dans les dallettes pour réaliser des dosages de concentration en chlorures à différentes profondeurs dans le béton.

Les profils de pénétration des chlorures obtenus montrent que la nappe de coffrage drainante a une influence significative sur la cinétique de pénétration des chlorures dans l'enrobage (figures 6 et 7) Elle a un rôle favorable pour la protection du béton d'enrobage contre ce phénomène à l'origine de la corrosion des armatures.

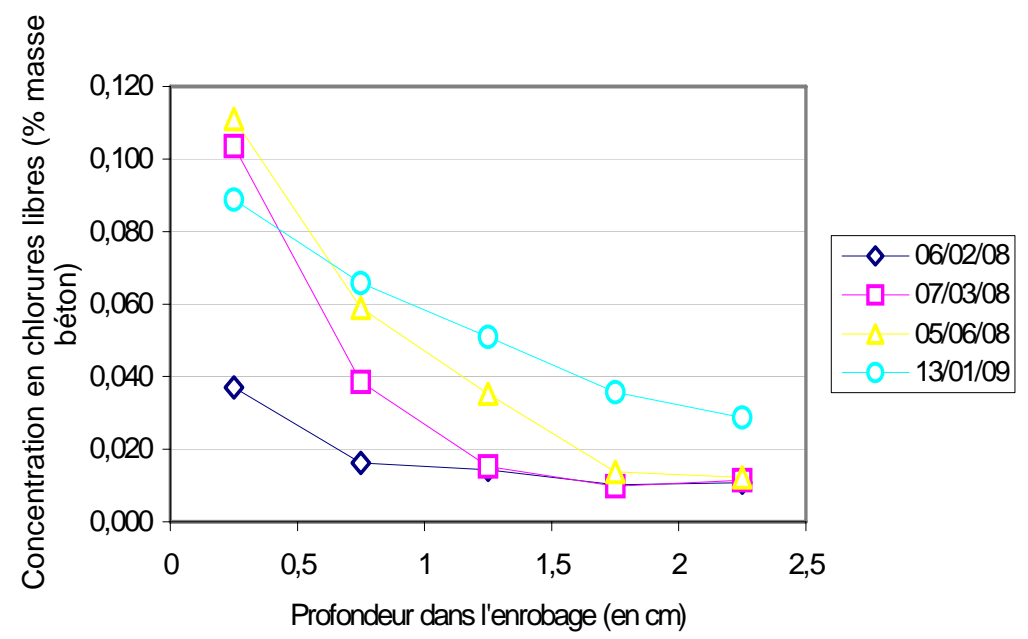

Figure 6. Profil de pénétration des chlorures - Dallette Cl (sans nappe). 


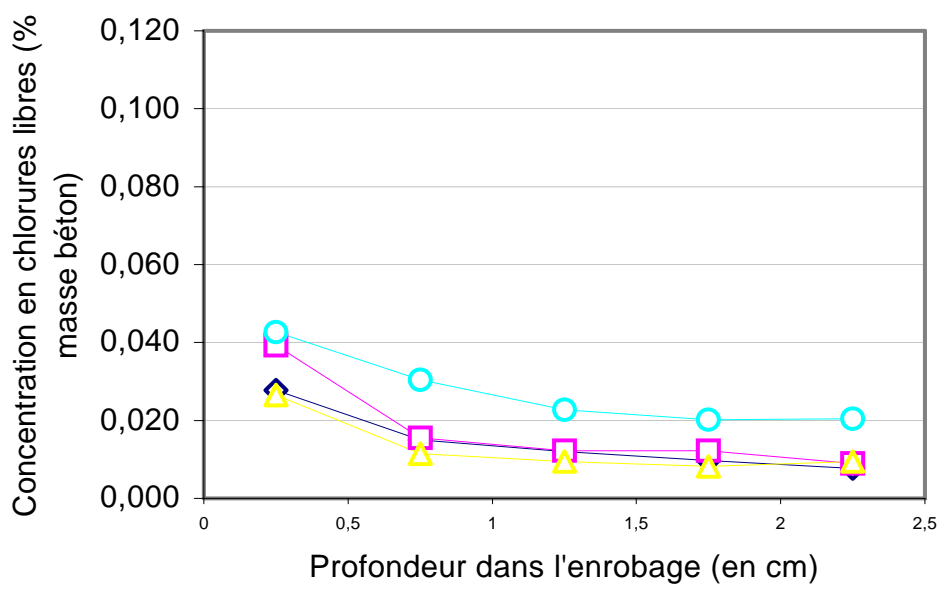

Figure 7. Profil de pénétration des chlorures - Dallette ZCl (avec nappe).

L'expérimentation a duré 454 jours. Le système de marnage a permis d'accélérer le phénomène de pénétration des chlorures par rapport à des conditions réelles d'exposition (environ 20 fois). Au terme de l'expérimentation, ces derniers ont pénétré jusqu'à environ $2 \mathrm{~cm}$ de profondeur. Au terme des 454 jours d'exposition (correspondant à environ 25 ans d'exposition en condition réelle), les écarts de concentration en chlorures entre la dallette "avec nappe" et la dallette "sans nappe" sont représentés à la figure 8 .

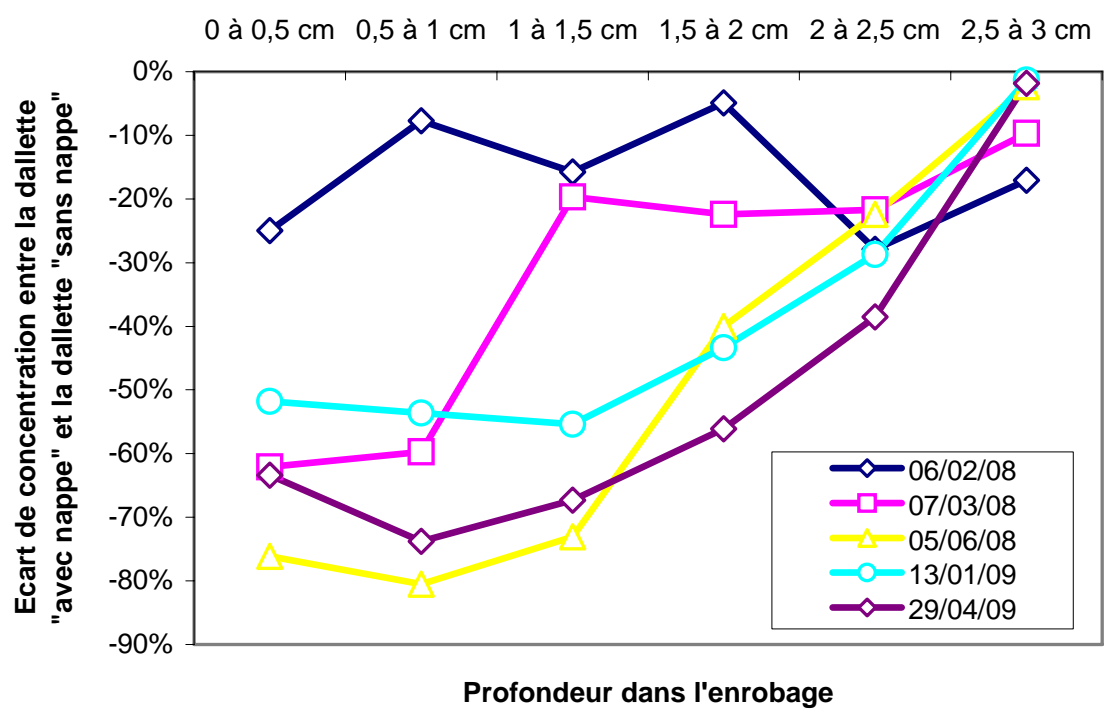

Figure 8. Ecart de concentration entre la dallette avec nappe et la dallette sans nappe. 
La mise en place d'un dispositif de nappe de coffrage drainante au moment de la construction d'une structure en béton armé contribue à ralentir sensiblement le phénomène de pénétration des chlorures dans les premiers centimètres du béton. Il a ainsi été constaté, au terme de l'expérimentation, une diminution de $60 \%$ de la concentration en chlorures dans les deux premiers centimètres. Le procédé a donc un effet sensible et favorable sur la durabilité de la structure vis-à-vis des risques de corrosion.

\section{Conclusion et perspectives}

La corrosion est l'une des principales causes de dégradation des ouvrages en béton armé. En environnement portuaire et maritime, la corrosion des armatures due à la pénétration des ions chlorures dans l'enrobage constitue la problématique majeure, notamment quand il s'agit de garantir une durabilité maximale des infrastructures.

Les nappes de coffrage drainantes sont mises en place sur les panneaux de coffrage avant bétonnage. Lors de la mise en œuvre du béton et plus tard lors de sa prise, elles drainent l'excès d'eau dans le béton à proximité du coffrage et assure une première phase de cure efficace. Elles améliorent ainsi, après la prise du béton, ses propriétés de transfert et plus précisément sa capacité à résister à la pénétration des chlorures.

L'objectif de l'étude était de confirmer les améliorations apportés par ce procédé et de les quantifier. Pour cela des corps d'épreuve ont été confectionnés et un dispositif de vieillissement accéléré a été conçu. A noter que ce dispositif a permis d'accélérer d'environ vingt fois le processus de pénétration des chlorures par rapport à des conditions réelles d'exposition aux cycles naturels de marnage. Autrement dit, les 454 jours d'expérimentation ont simulé un béton exposé au marnage pendant approximativement 25 ans.

L'étude a montré d'une part que les propriétés de transfert du béton d'enrobage mesurées sur les corps d'épreuve confectionnés avec la nappe de coffrage drainante étaient sensiblement améliorées sur les deux premiers centimètres de l'enrobage. L'étude a montré d'autre part que le procédé avait un impact significatif sur les profils de pénétration des chlorures. Les dernières campagnes de mesure ont cependant montré que cet impact semblait s'estomper avec le temps. La faible durée de l'expérimentation ne permet malheureusement pas de conclure sur ce point.

Au regard des résultats de l'étude, il semble pertinent d'émettre un avis favorable à l'utilisation du procédé de nappe de coffrage drainante pour les structures en béton armé en environnement maritime. Cet avis est néanmoins conditionné d'une part par le strict respect des conditions de mise en œuvre du procédé et d'autre part par une étude préalable de compatibilité du système avec les dispositions constructives. A titre anecdotique, l'intérêt du procédé pour les pièces bétonnées sous l'eau est discutable puisque l'effet drainant de la nappe est empêché par l'eau de mer présente en permanence durant la phase de bétonnage et de maturation du béton. 
Thème 5 - Ouvrages portuaires, côtiers et offshore

\section{Références Bibliographiques}

AFGC (2004). Conception des bétons pour une durée de vie donnée des ouvrages, maitrise de la durabilité vis à vis de la corrosion des armatures et de l'alcali-réaction. Documents scientifiques et techniques. 